Corrected: Author correction

ARTICLE

DOI: $10.1038 /$ s41467-017-01238-4

\title{
Nanoscale control of competing interactions and geometrical frustration in a dipolar trident lattice
}

\author{
Alan Farhan (1) ${ }^{1}$, Charlotte F. Petersen ${ }^{2}$, Scott Dhuey ${ }^{3}$, Luca Anghinolfi ${ }^{4}$, Qi Hang Qin ${ }^{5}$, Michael Saccone ${ }^{6}$, \\ Sven Velten ${ }^{7,8}$, Clemens Wuth ${ }^{9,10}$, Sebastian Gliga ${ }^{11}$, Paula Mellado ${ }^{12}$, Mikko J. Alava ${ }^{2}$, Andreas Scholl ${ }^{1}$ \\ \& Sebastiaan van Dijken ${ }^{5}$
}

Geometrical frustration occurs when entities in a system, subject to given lattice constraints, are hindered to simultaneously minimize their local interactions. In magnetism, systems incorporating geometrical frustration are fascinating, as their behavior is not only hard to predict, but also leads to the emergence of exotic states of matter. Here, we provide a first look into an artificial frustrated system, the dipolar trident lattice, where the balance of competing interactions between nearest-neighbor magnetic moments can be directly controlled, thus allowing versatile tuning of geometrical frustration and manipulation of ground state configurations. Our findings not only provide the basis for future studies on the low-temperature physics of the dipolar trident lattice, but also demonstrate how this frustration-by-design concept can deliver magnetically frustrated metamaterials.

\footnotetext{
${ }^{1}$ Advanced Light Source, Lawrence Berkeley National Laboratory (LBNL), 1 Cyclotron Road, Berkeley, CA 94720, USA. ${ }^{2}$ COMP Centre of Excellence, Department of Applied Physics, Aalto University, P.O. Box 11100, Espoo, Fl-00076 Aalto, Finland. ${ }^{3}$ Molecular Foundry, Lawrence Berkeley National Laboratory (LBNL), 1 Cyclotron Road, Berkeley, CA 94720, USA. ${ }^{4}$ Dipartimento di Fisica, Università di Genova, via Dodecaneso 33, I-16146 Genova, Italy. ${ }^{5}$ NanoSpin, Department of Applied Physics, Aalto University School of Science, P.O. Box 15100, FI-00076 Aalto, Finland. ${ }^{6}$ Department of Physics, University of California, Santa Cruz, CA 95064, USA. ${ }^{7}$ Materials Sciences Division, Lawrence Berkeley National Laboratory, 1 Cyclotron Road, Berkeley, CA 94720, USA. ${ }^{8}$ Institut für Nanostruktur- und Festkörperphysik, Universität Hamburg, Jungiusstrasse 11, 20355 Hamburg, Germany. ${ }^{9}$ Center for X-ray Optics, Lawrence Berkeley National Laboratory, 1 Cyclotron Road, Berkeley, CA 94720, USA. ${ }^{10}$ Daegu Gyeongbuk Institute of Science and Technology (DGIST), 50-1 Sang-ri, Hyeonpung-myeon, Dalseong-gun, Daegu 42988, Republic of Korea. ${ }^{11}$ SUPA, School of Physics and Astronomy, University of Glasgow, Glasgow G12 8QQ, UK. ${ }^{12}$ School of Engineering and Sciences, Adolfo Ibáñez University, Diagonal Las Torres 2640, Peñalolén, Santiago, Chile. Correspondence and requests for materials should be addressed to A.F. (email: alan.farhan@gmx.net)
} 
A rtificial square ice ${ }^{1}$, consisting of dipolar coupled Ising-type nanomagnets, lithographically arranged onto a two-dimensional square lattice, was introduced as a two-dimensional analog to pyrochlore spin ice ${ }^{2}$ and provided the prospect to directly explore the consequence of geometrical frustration using appropriate imaging techniques ${ }^{3-7}$. However, due to imbalanced competing interactions of nanomagnets meeting at the four-nanomagnet vertices, the spin-ice degeneracy in artificial square ice is lifted ${ }^{8}$ and the system lacks the macroscopic degeneracy of its three-dimensional counterpart, the pyrochlore spin ice. Indeed, artificial square ice, when exhibiting thermally driven moment fluctuations, has been shown to have a clear pathway toward a long-range ordered ground state configuration $^{9-12}$. Several concepts have been proposed to overcome this problem, most prominently by introducing a height offset between the corresponding nanomagnets ${ }^{8,13-15}$. However, a realization that is at the same time technically simple and appropriate for direct real-time imaging of thermal fluctuations remains elusive ${ }^{15}$. Alternatively, highly frustrated artificial kagome spin ice has been extensively investigated, as it exhibits some analogy to pyrochlore spin ice ${ }^{16}$, including a spin liquid phase with short-range correlations ${ }^{17-21}$. Still, long-range dipolar interactions have been shown to overcome the fixed degree of frustration at low-temperature regimes, leading the system to access a long-range ordered ground state ${ }^{17-19}$.

This raises the question whether a two-dimensional geometrical concept can be proposed that shares some similarities to the square ice geometry, while exhibiting a higher degree of geometrical frustration.

In the following, we address this point by exploring moment configurations achieved in a two-dimensional artificial frustrated system consisting of nanomagnets occupying the sites of a so-called trident lattice. Following thermal annealing, we observe how accessible low-energy configurations can be directly manipulated by tuning the balance of competing interactions.

\section{Results}

The dipolar trident lattice. We introduce an artificial frustrated system consisting of three-nanomagnet (trident) building blocks periodically arranged in a perpendicular fashion (Fig. 1a). Each nanomagnet is small enough to be single-domain and elongated, so that the magnetization points toward one of two possible directions along the long axis of each nanomagnet, thus representing a single Ising-type moment. As these moments couple via dipolar magnetic fields, we refer to this system as the dipolar trident lattice. Using synchrotron-based photoemission electron microscopy PEEM ${ }^{22}$ (Methods section), we directly visualize thermally induced magnetic relaxation of the trident lattice, and demonstrate the inability of the system to access a fully ordered state down to temperatures around $150 \mathrm{~K}$, when tuning the balance of competing interactions. We show how, above $150 \mathrm{~K}$, the ordering preferences of the system can be altered between two long-range ordered phases via an intermediate disordered state, exhibiting a continuous presence of vertex defects, which through their migration control the relaxation process and configurational fluctuations in thermal equilibrium. Upon cooling, the disordered phase also evolves toward long-range order, exhibiting a mixture of the two magnetic configurations.

Energetically, moment configurations in the trident lattice (Fig. 1b) can be characterized by four vertex types ${ }^{1,9}$ listed with increasing dipolar energy in Fig. 1c. In addition to the vertex types, the so-called trident types need to be taken into account, which are listed with increasing energy as Type A, B, and C in Fig. 1c. In order to understand the concept of competing interactions in the trident lattice, one has to be aware of the consequence of dipolar interactions: First, at four-nanomagnet vertices (for example, $\alpha, \delta$, $\varepsilon$, and $\eta$ in Fig. 1a), nearest neighbors will preferably exhibit a head-to-tail moment alignment, giving rise to Type I vertices (Fig. 1c) and vortex-like states (Fig. 1d). Second, the tridents $(\alpha, \beta$, and $\gamma$ in Fig. 1a) favor an anti-parallel moment alignment (Fig. 1d). In a long-range picture, a system where vertex interactions are mostly minimized (Type I vertex domination) cannot satisfy all trident interactions, as Type B tridents will dominate the configuration landscape (Fig. 1e). In contrast, if trident interactions are minimized (Type A trident domination), the energetically higher Type II vertices will exhibit a dominating presence (Fig. 1f). In other words, it is impossible to

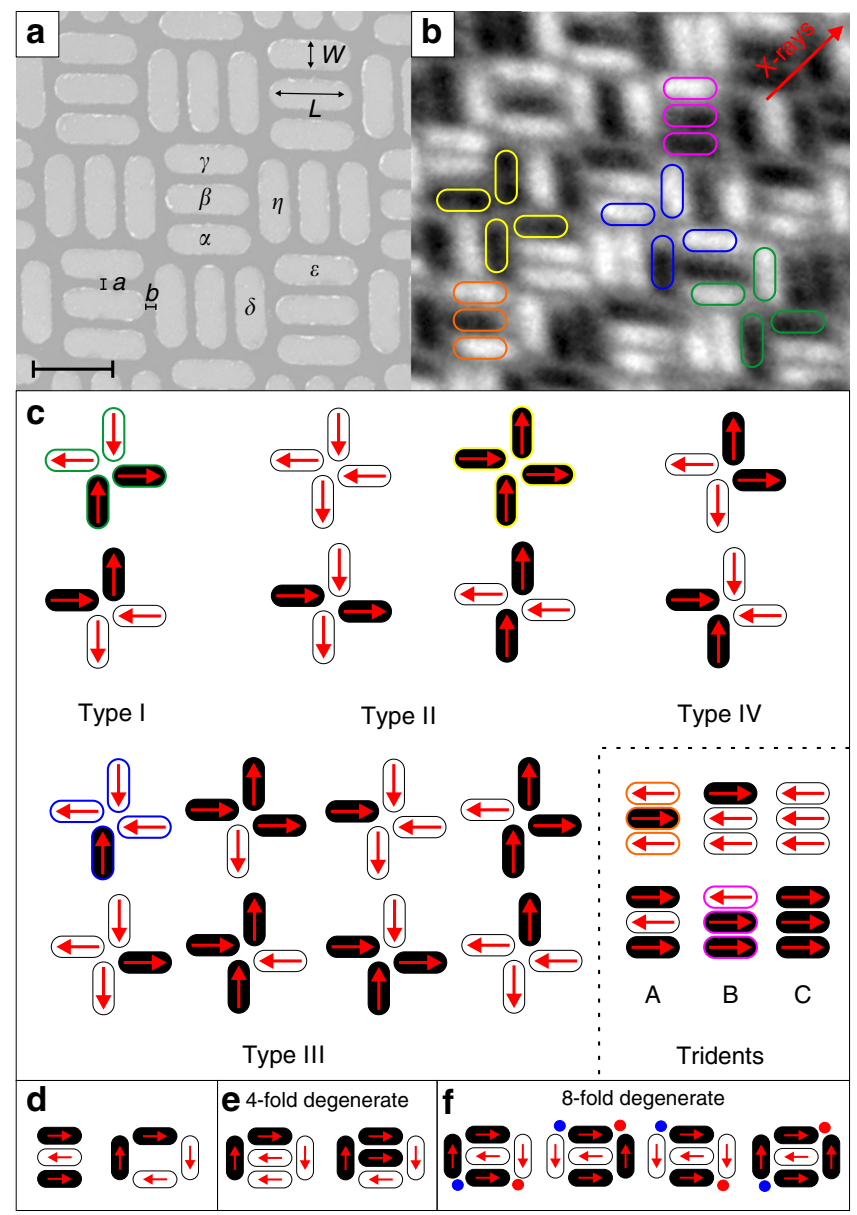

Fig. 1 The dipolar trident lattice. a Scanning electron microscope image of a dipolar trident lattice $(a=b=50 \mathrm{~nm}, L=450 \mathrm{~nm}, W=150 \mathrm{~nm})$. The black scale bar indicates a length of $450 \mathrm{~nm}$. b X-ray magnetic circular dichroism (XMCD) image resolving moment configurations achieved in the trident lattice. Nanomagnets with a magnetization pointing toward the incoming $X$ ray propagation vector (indicated by a red arrow) appear dark, while moments opposing that direction appear bright. c Vertex and trident types listed with increasing energy. In both $\mathbf{b}$, c green, yellow, and blue frames highlight the realizations of Type I, Type II, and Type III vertices, respectively. Regarding tridents, Type A and B tridents are highlighted with orange and magenta frames, respectively. $\mathbf{d}$ Minimization of dipolar interactions in an isolated trident building block would result in a Type A domination. On the other hand, nearest-neighbor nanomagnets at the fournanomagnet vertices will prefer a head-to-tail moment alignment, which would result in clockwise or anti-clockwise vortices. e Satisfying vertex interactions (creation of vortices or Type I vertices) results in frustration of trident moments. $\mathbf{f}$ Satisfying trident interactions (creation of Type A tridents) results in two of nearest-neighbor vertex moments being aligned head-to-head (red circles) or tail-to-tail (blue circles) 

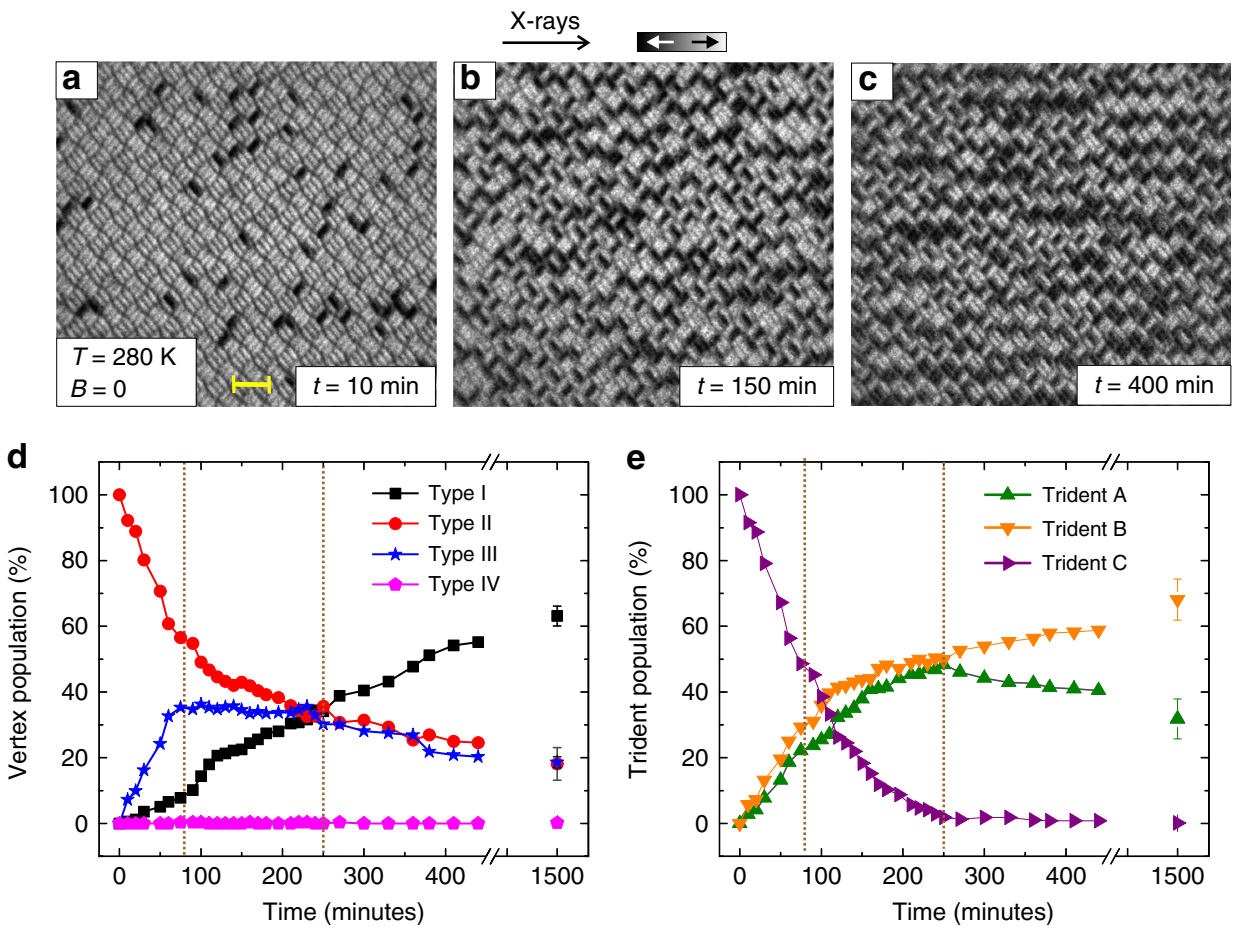

Fig. 2 Thermal relaxation of the dipolar trident lattice. a-c XMCD images of the dipolar trident lattice undergoing thermally induced magnetic relaxation from a magnetically saturated state to a low-energy equilibrium configuration. The yellow scale bar indicates a length of $1 \mu \mathrm{m}$. $\mathbf{d}$ Experimentally obtained temporal evolution of vertex-type population extracted from XMCD images recorded at a constant temperature $(T=280 \mathrm{~K})$. e Trident population plotted for the same relaxation process

simultaneously satisfy both vertex interactions and trident interactions and the system is expected to be frustrated.

Direct observation of thermal relaxation. As a first step, we aim to visualize the consequence of geometrical frustration on the ordering mechanism in a trident lattice consisting of nanomagnets with length, width, and thickness of 450, 150, and $2.7 \mathrm{~nm}$, respectively (Methods section). The lattice spacing was chosen, so that the two relevant parameters $a$ and $b$ (Fig. 1a), which control the strength of trident and vertex interactions, respectively, are set to be $50 \mathrm{~nm}$ each. The blocking temperature $T_{\mathrm{B}}$, which we define as the temperature at which moment reorientations start to occur within the timescale needed to acquire a single-PEEM image (7-9 s per image $)^{9,23}$ was determined to be $270 \mathrm{~K}$. The sample was kept at a constant temperature of $280 \mathrm{~K}$ and a saturating magnetic field $(B=30 \mathrm{mT})$ was shortly applied along the incoming X-ray direction. After the field is switched off, the system undergoes thermally induced magnetic relaxation from a well-defined energetically excited state toward a highly disordered equilibrium state (Fig. 2a-c; Supplementary Fig. 1 and Supplementary Movie 1).

A quantitative analysis of the relaxation mechanism is obtained by looking at the vertex-type and trident-type populations plotted as a function of time (Fig. 2d, e). Starting from a 100\% Type II vertex and Type $\mathrm{C}$ trident background (saturated state), the system experiences a rapid drop and rise in Type II and Type III vertex populations, respectively, while Type I vertices are moderately on the rise (Fig. $2 \mathrm{~d}$ ). In parallel, the population of Type C tridents decreases rapidly, while Type A and B tridents are showing an almost equal increase (Fig. 2e). As the system continues to relax, the high number of generated Type III vertex defects converts into Type I vertices, while new defects are continuously generated with an ongoing decrease in Type II vertices. Thus, the system stagnates in terms of Type III population during this stage (Fig. 2d). Finally, the system enters a stage where the Type I vertex population rises continuously at the cost of Type II and Type III vertices, until equilibrium is achieved. Kinetic Monte Carlo simulations (Methods section) ${ }^{19}$ are in good agreement with the experimental observations (Supplementary Fig. 2).

Controlling the balance of competing interactions. The dominance of Type I vertices for $a=b=50 \mathrm{~nm}$ indicates that the competition between vertex interactions and trident interactions is not perfectly balanced and, as a result, a high degree of frustration is not obtained. This balance of competing interactions can be tuned by varying the $b / a$ ratio. Therefore, a second set of trident arrays are fabricated (Methods section), where $a=50 \mathrm{~nm}$ is set to stay constant, while $b$ is varied to be 50,75 , and $100 \mathrm{~nm}$. The sample was kept at a constant temperature of $330 \mathrm{~K}$ $\left(T_{\mathrm{B}}=310 \mathrm{~K}\right)$ for $\sim 24 \mathrm{~h}$ before it was cooled down to $300 \mathrm{~K}$ and magnetic images were obtained (Fig. 3a-c; Supplementary Fig. 3). Plotting the vertex populations and trident populations as a function of $b$ (Fig. 3d, e), we see a transition from a largely ordered phase with Type I vertex and Type B trident domination $(b=50 \mathrm{~nm})$, through a disordered phase with short-range order and no clear preference for any vertex types $(b=75 \mathrm{~nm})$, to, finally, a phase that shows trends toward Type II vertex and Type A trident preference $(b=100 \mathrm{~nm})$.

This balancing act between competing trident interactions and vertex interactions indicates that accessible low-energy states can be directly tuned by a variation of the $b / a$ ratio. This is a direct consequence of the enforced lattice constraints, making it impossible for the involved entities to simultaneously minimize both trident interactions and vertex interactions. Similar to previous work ${ }^{24,25}$, calculating the energy spectrum of an isolated five-nanomagnet building block (Supplementary Fig. 4), clarifies the degeneracies listed in Fig. 1e, f. For a system with $b / a=1$, the 

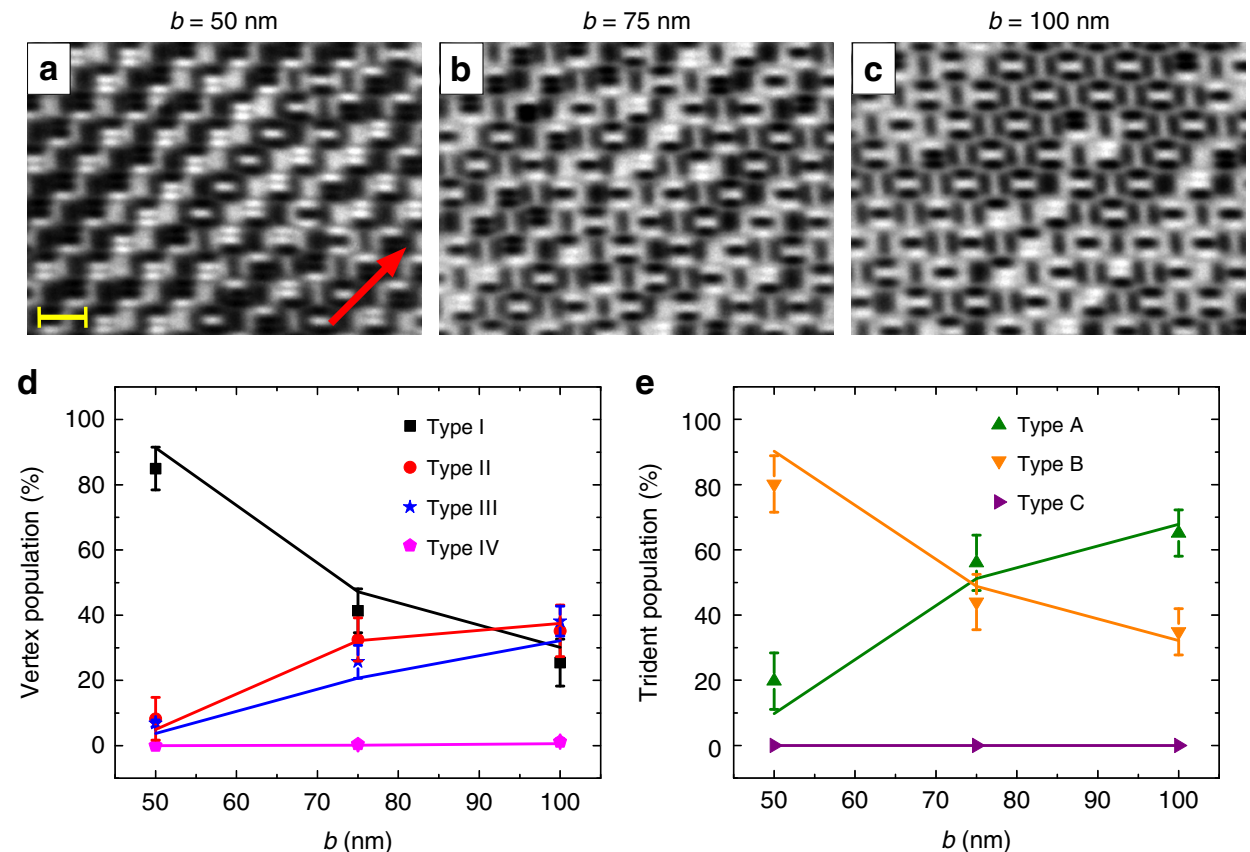

Fig. 3 Tuning geometrical frustration in dipolar trident lattice. a-c XMCD images of equilibrium configurations of trident lattices with various lattice spacings $\left(a=50 \mathrm{~nm}=\right.$ const, $b=50,75$, and $100 \mathrm{~nm}$ ) recorded at $T=300 \mathrm{~K}$ (blocking temperature $T_{\mathrm{B}}=310 \mathrm{~K}$ ). The red arrow indicates the direction the $\mathrm{X}$-ray propagation vector. The yellow scale bar indicates a length of $1 \mu \mathrm{m}$. $\mathbf{d}$ Experimentally observed vertex-type population plotted as a function of lattice parameter $b$. e Trident-type population plotted with increasing lattice parameter $b$. Experimental observations (filled symbols) are in satisfactory agreement with equilibrium Monte Carlo simulations (lines). The results reveal the transition from a Type I vertex and Type B trident-ordered state $(b=50 \mathrm{~nm})$, to a disordered configuration ( $b=75 \mathrm{~nm}$ ) with no clear ordering preference, and, finally, to a Type A trident-ordered state $(b=100 \mathrm{~nm})$, as the lattice parameter $b$ is increased. The error bars represent standard deviations originating from ten experimental observations

$b=33 \mathrm{~nm}$

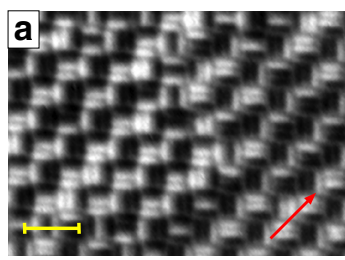

d

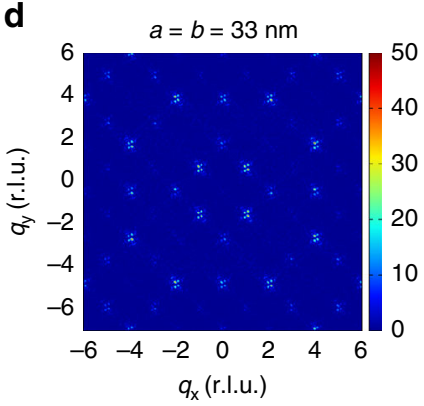

$b=50 \mathrm{~nm}$

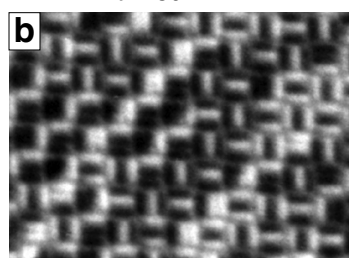

e

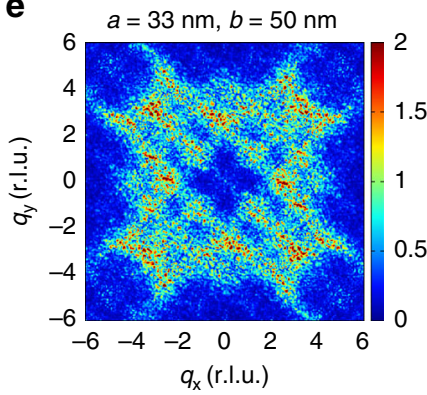

$b=66 \mathrm{~nm}$

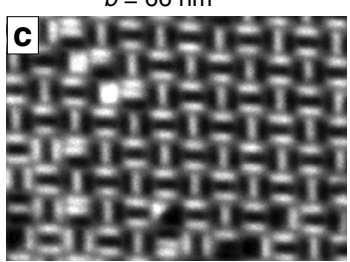

f

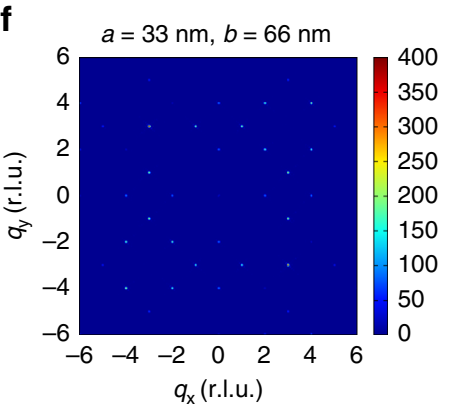

Fig. 4 Experimental low-temperature observations. a-c XMCD images of equilibrium configurations of the dipolar trident lattice with various lattice spacings ( $a=33 \mathrm{~nm}=$ const, $b=33,50$, and $66 \mathrm{~nm}$ ) recorded at $T=150 \mathrm{~K}$ (blocking temperature $T_{\mathrm{B}}=160 \mathrm{~K}$ ). The red arrow indicates the direction the $X$-ray propagation vector. The yellow scale bar indicates a length of $\sim 600 \mathrm{~nm}$. d-f Magnetic scattering patterns of moment configurations achieved in dipolar trident lattices with lattice parameters $\mathbf{d} a=33 \mathrm{~nm}$ and $b=33 \mathrm{~nm}$, e $a=33 \mathrm{~nm}$ and $b=50 \mathrm{~nm}$, and $\mathbf{f} a=33 \mathrm{~nm}$ and $b=66 \mathrm{~nm}$, following thermal annealing. While long-range order with relatively sharp peaks is seen for $b=33 \mathrm{~nm}$ and $b=66 \mathrm{~nm}$, the diffuse patterns for $b=50 \mathrm{~nm}$ indicate highly disordered moment configurations with short-range correlations

building block ground state is four-fold degenerate, with a clear gap ( $\Delta E$ in Supplementary Fig. 4$)$ to the second energy band, which consists of eight quasi-degenrate states. This gap can be tuned by varying the $b / a$ ratio. The critical ratio $b / a=1.5$ of the relevant lattice parameters can be further comprehended, when comparing the dipolar energies for fully ordered magnetic configurations of Type A/Type II and Type B/Type I trident types and vertex types as a function of $b$, while $a$ is kept constant at $50 \mathrm{~nm}$. This is shown in Supplementary Fig. 5, where dipolar energies are equalized, when $b$ reaches a value around $75 \mathrm{~nm}$. 
In other words, this is the point where the dipolar trident lattice reaches maximum degeneracy with no preference for any of the twelve states listed in Fig. 1e, f.

Low-temperature configurations and magnetic structure factors. As a next step, we study how the degeneracy of low-energy building block states affects moment configurations at lower temperatures. Previous work on highly frustrated artificial kagome spin ice $\mathrm{e}^{17,24,26}$ showed that despite an extensive degeneracy and short-range ordering at higher temperatures, the long-range nature of dipolar interactions gives rise to ordered configurations at lower temperatures. Therefore, it is our purpose here to see whether any signatures of long-range ordering can be observed in the dipolar trident lattice, particularly in the case of highest degeneracy, when $b / a=1.5$.

We prepared another set of trident lattices, consisting of nanomagents with lengths $L=300 \mathrm{~nm}$, widths $W=100 \mathrm{~nm}$, and thickness $d=2.4 \mathrm{~nm}$, together with the corresponding lattice parameters $a=33 \mathrm{~nm}=$ const. and $b=33,50$, and $66 \mathrm{~nm}$. This reduction of nanomagnet size resulted in a lowering of the blocking temperature down to $160 \mathrm{~K}$. The sample was kept in vacuum at room temperature for 20 days, before it was cooled down to $150 \mathrm{~K}$ for X-ray magnetic circular dichroism (XMCD) image acquisition (Fig. 4a-c). While long-range ordered patterns are observed for $b=33$ and $66 \mathrm{~nm}$, the $b=50 \mathrm{~nm}$ array remains disordered at $150 \mathrm{~K}$. Similar to the room-temperature data (Fig. 3), tuning of geometrical frustration can again be inferred from the evolution of vertex populations and trident populations as a function of lattice parameter $b$ (Supplementary Fig. 6). Our results thus indicate that for the case $b / a=1.5$, the system is caught in a short-range ordered phase, while both the $b / a=1$ and 2 cases exhibit long-range ordered ground state configurations.

A deeper quantitative insight into the experimentally accessed low-temperature configurations is achieved by calculating the respective magnetic structure factors ${ }^{15}$ (Methods section), which are shown in Fig. $4 \mathrm{~d}-\mathrm{f}$. For both $b=33 \mathrm{~nm}$ and $b=66 \mathrm{~nm}$ (Fig. 4d, f), we see relatively sharp peaks in the magnetic structure factors. The splitting of the magnetic peaks into four satellites for $b=33 \mathrm{~nm}$ reflects multi-domain long-range ground state ordering for $b=33 \mathrm{~nm}$ (Fig. $4 \mathrm{~d})$. Figure $4 \mathrm{f}(\mathrm{b}=66 \mathrm{~nm})$ shows sharp magnetic peaks that stand for an almost single-domain longrange ordered ground state consisting mostly of a tile of two Type A tridents, as can also be seen from real-space images (Fig. 4c). However, a dramatic change in the magnetic structure factor is observed for the lattice parameter combination of $a=33 \mathrm{~nm}$ and $b=50 \mathrm{~nm}$ (Fig. 4e), where the diffuse patterns indicate the presence of a disordered phase consisting of a complex arrangements of possible low-energy configurations (Fig. 1e, f), where neither of these states dominate. Similar patterns are also observed in the structure factor of the simulated $b=50 \mathrm{~nm}$ system (Supplementary Fig. 7). To ensure that these simulated configurations are in fact in thermal equilibrium, we use the parallel tempering technique ${ }^{27-29}$ (Methods section), where the equilibration time is estimated by calculating the so-called exponential autocorrelation time ${ }^{29}, \tau_{\exp }$, which itself is defined by the temporal decay of the autocorrelation function, $\Gamma \propto \exp$ $\left[-t / \tau_{\exp }\right]$ (Methods section). The agreement between experimental and simulated configurations provides evidence that the experimental observations also represent states in thermal equilibrium. For comparison, we also calculated the magnetic structure factor of a purely paramagnetic trident lattice (Supplementary Fig. 8). In this case, no peaks appear in the structure factor map, providing further evidence that our dipolar trident lattice with $a=33 \mathrm{~nm}$ and $b=50 \mathrm{~nm}$ is in a disordered phase, where none of the predicted ground states is able to dominate the landscape at $150 \mathrm{~K}$. While only partial order is able to set in at $150 \mathrm{~K}$ with relatively weak peak intensities, magnetic structure factor simulations clearly show that the peak intensities rise with decreasing temperature (Supplementary Fig. 9). Eventually, the system evolves toward long-range order at low temperatures, exhibiting a mixture of ordered stripes (Supplementary Fig. 10), which consist of a tile of Type A and Type B tridents and a mixture of Type I and Type II vertices. Compared to classical artificial square ice, which exhibits trivial ground state ordering, the mixed phase patterns in the dipolar trident lattice reflect the high degree of frustration in this geometrically frustrated magnetic metamaterial. For interested readers, a comparison of experimental observations to artificial square ice can be found in Supplementary Note 1.

\section{Discussion}

In summary, we presented a magnetically frustrated metamaterial, which provides the possibility to directly control competing dipolar interactions at the nanoscale, thus allowing versatile tuning of geometrical frustration and ground state configurations. The complex phase into which the system gets trapped, when competing interactions are balanced, opens up multiple questions regarding the physics of the dipolar trident lattice, in particular the question regarding possible phase transitions toward complex long-range ordered states at lower temperature regimes ${ }^{17-19}$. Experimentally, this will require the fabrication of trident lattices consisting of nanomagnets with lateral dimensions that go beyond the spatial resolution of known magnetic imaging techniques ${ }^{17}$, and will therefore rely on emerging scattering and spectroscopic techniques ${ }^{17}, 26,30,31$.

\section{Methods}

Sample fabrication. Similar to previous work ${ }^{5,23}$, dipolar trident lattices were fabricated by taking advantage of lift-off-assisted electron-beam lithography: a silicon (100) substrate was first spin-coated with a 70-nm-thick layer of polymethylmethacrylate resist. Then, trident lattices with various lattice spacings were defined onto the sample with a VISTEC VB300 electron beam writer. Next, using a Semicore SC600 e-beam evaporator, a ferromagnetic permalloy $\left(\mathrm{Ni}_{80} \mathrm{Fe}_{20}\right)$ film was deposited at a base pressure of $1.2 \times 10^{-7}$ Torr, which was followed by lift-off in acetone at a temperature of $50^{\circ} \mathrm{C}$. Thermally driven moment fluctuations in one set of artificial spin ice samples were realized by fabrication of ultrathin nanomagnets with length $L=450 \mathrm{~nm}$ and width $W=150 \mathrm{~nm}$. The samples discussed in this work had thicknesses of $2.7 \mathrm{~nm}$ and $3 \mathrm{~nm}$, resulting in blocking temperatures of 270 and $310 \mathrm{~K}$, respectively. For low-temperature measurements, the blocking temperature was moved down to $160 \mathrm{~K}$ by preparing nanomagnets with lengths, widths, and thickness of 300,100 , and $2.4 \mathrm{~nm}$, respectively.

Photoemission electron microscopy. Measurements were performed using the cryogenic photoemission electron microscope PEEM3 at beamline 11.0.1 at the Advanced Light Source ${ }^{22}$. Magnetic images were captured by taking advantage of $\mathrm{XMCD}$ at the $\mathrm{Fe}_{3}$-edge ${ }^{32}$. The obtained contrast is a measure of the projection of the magnetization on the X-ray polarization vector, so that nanomagnets with a magnetization parallel or antiparallel to the X-ray polarization either appear black or white. Nanomagnets with moments having $\pm 45^{\circ}$ and $\pm 135^{\circ}$ angles with respect to the incoming X-rays appear dark and bright, respectively.

Magnetic structure factor. The magnetic structure factor is calculated as

$$
I(\mathbf{q})=\frac{1}{N} \sum_{i=1}^{N} \sum_{j=1}^{N} \mathbf{S}_{i}^{\perp} \cdot \mathbf{S}_{j}^{\perp} \exp \left(i \mathbf{q} \cdot \mathbf{r}_{i, j}\right),
$$

where $\mathbf{S}_{i}^{\perp}=\mathbf{S}_{i}-\left(\widehat{\mathbf{q}} \cdot \mathbf{S}_{i}\right) \widehat{\mathbf{q}}$ is the component of the spin vector of each island perpendicular to the reciprocal space vector $\mathbf{q}$, the unit vector is given by $\widehat{\mathbf{q}}=\mathbf{q} /\|\mathbf{q}\|, \mathbf{r}_{i, j}$ is the vector from island $i$ to $j$, and $N$ is the total number of islands. Equation (1) has the same form as in neutron scattering experiments and has previously been used to analyze artificial spin-ice configurations ${ }^{15}$.

Simulations. We model each nanomagnet as an infinitesimally thin compass needle with a uniform magnetic moment density $\frac{|m|}{L}$. The magnetic moment points along the long axis of the island. This description is equivalent to placing a 
magnetic charge at each end of the island ${ }^{5,19,33}$. The inter-island interaction is given by the Hamiltonian

$$
H_{i j}=\frac{\mu_{0}|m|^{2}}{4 \pi L^{2}}\left[\frac{1}{\left|r_{a_{i}}-r_{a_{j}}\right|}-\frac{1}{\left|r_{a_{i}}-r_{b_{j}}\right|}-\frac{1}{\left|r_{b_{i}}-r_{a_{j}}\right|}+\frac{1}{\left|r_{b_{i}}-r_{b_{j}}\right|}\right],
$$

where $r_{a_{i}}$ and $r_{\mathrm{bi}}$ are the locations of the positive and negative magnetic charge on the $i$ th nanomagnet, $\mu_{0}$ is the magnetic permeability, $L$ is the island length, and $|m|$ $=M V$ is the magnetic moment of each nanomagnet with $M$ being the saturation magnetization and $V$ the nanomagnet volume. The system size is 1200 islands, and only interactions with a magnitude of at least $2 \%$ of the nearest-neighbor interaction are included in the simulation ( 35 neighbors per spin).

To simulate the dynamics of the system, we use the kinetic Monte Carlo method $^{9,23}$, which evolves the system through single-spin flips. A particular spin flip move is selected with a probability proportional to its rate.

Assuming an Arrhenius-type switching behavior, the rate of a spin flip is given by $v=v_{0} \exp \left(-E / k_{\mathrm{B}} T\right)$, where $k_{\mathrm{B}}=8.62 \times 10^{-5} \mathrm{eV} \mathrm{K}^{-1}$ is the Boltzmann constant, $\nu_{0}$ is the so-called attempt frequency, $T$ is the temperature, and $E$ is the reorientation barrier, which is equal to the intrinsic energy barrier $E_{0}$ plus half the dipolar energy gain associated with moment re-orientations (Eq. (2)). The simulation parameters $M=240 \mathrm{kA} \mathrm{m}^{-1}, E=0.887 \mathrm{eV}$, and $\nu_{0}=10^{12} \mathrm{~s}^{-1}$ were fit using the experimental relaxation results of Fig. 2. These values are in good agreement with previous studies on thermally activated artificial spin ice ${ }^{5,9}, 23$. In addition to the assumption of a uniform system, where all nanomagnets have the same intrinsic energy barrier, we also investigated the role of disorder ${ }^{9}$. This is included by assuming a random variation in $E_{0}$, which follows a Gaussian distribution with mean $E_{0}=0.893 \mathrm{eV}$ and standard deviation $\sigma=0.05 \mathrm{eV}$ (Supplementary Fig. 2).

To generate equilibrium configurations, for the results presented in Fig. 3 and structure factor calculations shown in Supplementary Fig. 7, we use the parallel tempering technique ${ }^{27,28}$. Replicas of the system are simulated at a number of temperatures simultaneously using kinetic Monte Carlo. After every Monte Carlo sweep a move is proposed which swaps the configuration of a pair of replicas at neighboring temperatures $T_{n}$ and $T_{m}$. This move is accepted with a probability

$$
P_{\text {swap }}(n, m)=\min \left\{1, \exp \left[-\left(\frac{1}{k_{\mathrm{B}} T_{m}}-\frac{1}{k_{\mathrm{B}} T_{n}}\right)\left(E_{n}-E_{m}\right)\right]\right\},
$$

where $E_{n}$ is the energy of replica $n$. The set of temperatures is selected such that the acceptance ratio of a swap move at each temperature is greater than 0.2 . A value of $M=362 \mathrm{kA} \mathrm{m}^{-1}$ is used to obtain the results in Fig. 3. The equilibration time is estimated with the exponential autocorrelation time ${ }^{29}, \tau_{\text {exp. }}$. This is defined by the decay of the autocorrelation function, $\Gamma \propto \exp \left[-t / \tau_{\exp }\right]$. It is calculated for the autocorrelation function of the spin overlap function between two concurrent independent simulations, its absolute value, and the configuration energy throughout the parallel tempering simulation. Taking the largest of these calculated exponential autocorrelation times, the first $20 \times \tau_{\exp }$ time steps are treated as equilibration time and discarded.

Code availability. Codes for numerical calculations in this study are available from the corresponding author upon reasonable request.

Data availability. Data supporting the findings in this study are available from the authors upon request.

Received: 18 October 2016 Accepted: 31 August 2017

\section{References}

1. Wang, R. F. et al. Artificial 'spin ice' in a geometrically frustrated lattice of nanoscale ferromagnetic islands. Nature 439, 303-306 (2006).

2. Bramwell, S. T. \& Gingras, M. J. P. Spin ice state in frustrated magnetic pyrochlore materials. Science 294, 1495-1501 (2001).

3. Heyderman, L. J. \& Stamps, R. L. Artificial ferroic systems: novel functionality from structure, interactions and dynamics. J. Phys. Condens. Matter 25, 363201 (2013).

4. Nisoli, C., Moessner, R. \& Schiffer, P. Colloquium: artificial spin ice: designing and imaging magnetic frustration. Rev. Mod. Phys. 85, 1473-1490 (2013).

5. Farhan, A. et al. Thermodynamics of emergent magnetic charge screening in artificial spin ice. Nat. Commun. 7, 12635 (2016).

6. Gilbert, I. et al. Emergent ice rule and magnetic charge screening from vertex frustration in artificial spin ice. Nat. Phys. 10, 670-675 (2014).

7. Gilbert, I. et al. Emergent reduced dimensionality by vertex frustration in artificial spin ice. Nat. Phys. 12, 162-165 (2016).

8. Möller, G. \& Moessner, R. Artificial square ice and related dipolar nanoarrays. Phys. Rev. Lett. 96, 237202 (2006).
9. Farhan, A. et al. Direct observation of thermal relaxation in artificial spin ice. Phys. Rev. Lett. 111, 057204 (2013).

10. Morgan, J. P., Stein, A., Langridge, S. \& Marrows, C. H. Thermal ground-state ordering and elementary excitations in artificial magnetic square ice. Nat. Phys. 7, 75-79 (2011).

11. Zhang, S. et al. Crystallites of magnetic charges in artificial spin ice. Nature $\mathbf{5 0 0}$ 553-557 (2013).

12. Porro, J. M., Bedoya-Pinto, A., Berger, A. \& Vavassori, P. Exploring thermally induced states in square artificial spin-ice arrays. New J. Phys. 15, 055012 (2013).

13. Chern, G.-W., Reichhardt, C. \& Nisoli, C. Realizing three-dimensional artificial spin ice by stacking planar nano-arrays. Appl. Phys. Lett. 104, 013101 (2014).

14. Thonig, D., Reißaus, S., Mertig, I. \& Henk, J. Thermal string excitations in artificial spin-ice square dipolar arrays. J. Phys. Condens. Matter 26, 266006 (2014).

15. Perrin, Y., Canals, B. \& Rougemaille, N. Extensive degeneracy, Coulomb phase and magnetic monopoles in artificial square ice. Nature 540, 410-413 (2016).

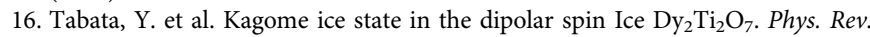
Lett. 97, 257205 (2006).

17. Anghinolfi, L. et al. Thermodynamic phase transitions in a frustrated magnetic metamaterial. Nat. Commun. 6, 8278 (2015).

18. Chern, G. W., Mellado, P. \& Tchernyshyov, O. Two-stage ordering of spins in dipolar spin ice on the kagome lattice. Phys. Rev. Lett. 106, 207202 (2011).

19. Möller, G. \& Moessner, R. Magnetic multipole analysis of kagome and artificial spin-ice dipolar arrays. Phys. Rev. B 80, 140409 (2009).

20. Canals, B. et al. Fragmentation of magnetism in artificial kagome dipolar spin ice. Nat. Commun. 7, 11446 (2016)

21. Chioar, I. A. et al. Kinetic pathways to the magnetic charge crystal in artificial dipolar spin ice. Phys. Rev. B 90, 220407 (2014).

22. Doran, A. et al. Cryogenic PEEM at the advanced light source. J. Electron Spectrosc. Relat. Phenom. 185, 340-346 (2012).

23. Farhan, A. et al. Exploring hyper-cubic energy landscapes in thermally active finite artificial spin-ice systems. Nat. Phys. 9, 375-382 (2013).

24. Farhan, A. et al. Thermally induced magnetic relaxation in building blocks of artificial kagome spin ice. Phys. Rev. B 89, 214405 (2014).

25. Mengotti, E. et al. Building blocks of an artificial kagome spin ice: Photoemission electron microscopy of arrays of ferromagnetic islands. Phys. Rev. B 78, 144402 (2008).

26. Sendetskyi, O. et al. Magnetic diffuse scattering in artificial kagome spin ice. Phys. Rev. B 93, 224413 (2016).

27. Earl, D. J. \& Deem, M. W. Parallel tempering: theory, applications, and new perspectives. Phys. Chem. Chem. Phys. 7, 3910-3916 (2005).

28. Swendsen, R. H. \& Wang, J.-S. Replica Monte Carlo simulation of spin-glasses. Phys. Rev. Lett. 57, 2607-2609 (1986).

29. Yucesoy, B., Machta, J. \& Katzgraber, H. G. Correlations between the dynamics of parallel tempering and the free-energy landscape in spin glasses. Phys. Rev. E 87, 012104 (2013).

30. Morley, S. A. et al. Vogel-Fulcher-Tammann freezing of a thermally fluctuating artificial spin ice probed by X-ray photon correlation spectroscopy. Phys. Rev. B 95, 104422 (2017).

31. Perron, J. et al. Extended reciprocal space observation of artificial spin ice with X-ray resonant magnetic scattering. Phys. Rev. B 88, 214424 (2013).

32. Stohr, J. W. et al. Magnetic microscopy with circularly polarized X-rays. Science 259, 658-661 (1993).

33. Castelnovo, C., Moessner, R. \& Sondhi, S. L. Magnetic monopoles in spin ice. Nature 451, 42-45 (2008).

\section{Acknowledgements}

We would like to thank Sujoy Roy, Guido Meier, Peter Fischer, Kevin Franke, and Zoe Budrikis for their support and fruitful discussions. This project was funded by the Swiss National Science Foundation and part of this work was performed at the Advanced Light Source and the Molecular Foundry, Lawrence Berkeley National Laboratory, 94720 Berkeley, USA. The Advanced Light Source and the Molecular Foundry are supported by the Director, Office of Science, Office of Basic Energy Sciences, of the U.S. Department of Energy under Contract No. DE-AC02-05CH11231. C.F.P. and M.J.A. are supported by the Academy of Finland through its Centres of Excellence Program (2012-2017) under project no. 251748 and the FiDiPro program, project 13282993. They acknowledge the computational resources provided by the Aalto University School of Science "Science-IT" project. S.G. was funded by the European Union's Horizon 2020 research and innovation programme under the Marie Sklodowska-Curie grant agreement No. 708674

\section{Author contributions}

A.F. designed this study and planned the experiments accordingly. A.F., S.D. and Q.H.Q. fabricated the samples. A.F., A.S. and S.G. performed XMCD imaging at the 
Advanced Light Source. A.F., L.A., C.F.P., M.S., S.V. and C.W. analyzed the obtained data. C.F.P., M.J.A. and P.M. provided the theoretical background to the study. A.S. and S.v.D. supervised the project. All authors contributed to the manuscript.

\section{Additional information}

Supplementary Information accompanies this paper at 10.1038/s41467-017-01238-4.

Competing interests: The authors declare no competing financial interests.

Reprints and permission information is available online at http://npg.nature.com/ reprintsandpermissions/

Publisher's note: Springer Nature remains neutral with regard to jurisdictional claims in published maps and institutional affiliations. (c) (i) Open Access This article is licensed under a Creative Commons Attribution 4.0 International License, which permits use, sharing, adaptation, distribution and reproduction in any medium or format, as long as you give appropriate credit to the original author(s) and the source, provide a link to the Creative Commons license, and indicate if changes were made. The images or other third party material in this article are included in the article's Creative Commons license, unless indicated otherwise in a credit line to the material. If material is not included in the article's Creative Commons license and your intended use is not permitted by statutory regulation or exceeds the permitted use, you will need to obtain permission directly from the copyright holder. To view a copy of this license, visit http://creativecommons.org/ licenses/by/4.0/.

(C) The Author(s) 2017 